\title{
Real time supply chain management: co-modeling of total supply chain solutions
}

\author{
Walid Bouzayani $^{1}$, Safwat Altal ${ }^{2}$, Mohamed M. Dhiaf ${ }^{3}$ \\ ${ }^{1}$ Computer and Embedded National Engineering School of Sfax, Tunisia \\ ${ }^{2}$ Emirates College of Technology United Arab Emirates \\ ${ }^{3}$ Faculty of Economics and Management of Sfax, Tunisia
}

Email address:

bouzayaniwalid2000@yahoo.fr(W. Bouzayani), Safwat.yousef@ect.ac.ae(S. Altal), mohamedmahjoub.dhiaf@fssegs.rnu.tn(M. M. Dhiaf)

\section{To cite this article:}

Walid Bouzayani, Safwat Altal, Mohamed M. Dhiaf. Real Time Supply Chain Management Co-Modeling of Total Supply Chain Solutions. Science Journal of Business and Management. Vol. 1, No. 1, 2013, pp. 8-13. doi: 10.11648/j.sjbm.20130101.12

\begin{abstract}
The diversification of collaborations and the complexity of processes in the same Supply Chain Management (SCM) needs a powerful and a flexible Information System (IS). For that, the classic modeling solutions of information systems cannot satisfy all the constraints of the complex E-SCM. Furthermore, the total E-SCM with the two brands of information system and SCM require advanced methods for co-modeling of complex systems. For more implication of all SCM actors, we consider an integration and dynamic solution for the management of supply chain activities a good tendency to achieve all industries challenges. This paper presents an approach for the total real time supply chain management. We adopt a method based on co-modeling of distributed Information System (IS) architecture in complex supply chain management.
\end{abstract}

Keywords: Real Time, Co-Modeling, Communication, Process, Supply Chain

\section{Introduction}

For decades, management solutions underwent many modifications in order to reply to industries requirements. The IS must manage with reliability all the processes and the actors from the supplier to the customer. The classic solutions of ERP cannot respond with efficiency to the procurement and the internal and external logistic activities[19]. In fact, the coordination between all actors and processes is still limited.

With great attention to improvement and growth, companies are always looking for the best solutions for monitoring and controlling its processes. The aim is to master the costs and delays in the first step, and to increase the satisfaction of their collaborations in a second step (customers, suppliers, banks, etc.).

Real-time process management is essentially based on architectures and communications technology. However, the IS that manages these processes have become increasingly complex. This is a consequence of the diversification of internal and external stakeholders, the different types of information required, in addition to the complexity of relationships. For this reason, the IS is seeking to meet and fulfill all these needs with the best triplet: Cost, Quality and Time.

With extensive development of wireless communication protocols, Ad hoc and mobile technology are often used in new systems. Despite the constraints on processing capacity, energy consumption, data processing and security, this technology continues to expand. with Wireless Networks, several areas gain benefits[1] with a remarkable evolution in the quality of services. Similarly, systems with a distributed architecture occupy places in critical new designs of ERP solutions. Despite the diversification of ERP systems, companies or organizations always try to adapt to their needs and specifications. Typically, these applications have standard modules for process management and supply chains of companies or organizations. Configuration does not lead often to meet exact needs. In addition, the time management in these solutions is limited which leads to a reduced flexibility. Hence, the automation of the internal and external supply chain management seems fundamental. However, the IS should be adopted to leverage the overall performance of the company[2]. Indeed, the collaboration of logistics activities between the different parts must be performed in 
good times to increase the service rates of various processes.

In this paper, we will adopt an approach based on operating and integrating wireless technologies (wireless sensor network WSN and Radio-frequency identification RFID)for the collection and processing of information in real-time solution ensuring better internal and external collaboration of all stakeholders of the total SCM.To respond to this objective, we will seek a flexible and mobile enabling real-time management of all processes in the supply chain of a company through a co-modeling activity of SCM and IS to converge to the expected results.

\section{Literature Review}

\subsection{Supply chain Management}

To ensure better communication within the SCM, A. Roberto and B. Glenn[7] proposed the Last Planner System (LPS) solution web-based techniques for time management information to enhance traditional methods of planning and scheduling in the production site. This strategy is based on a Logistic Center (LC) using a distributed production control tool which contains information and products from all suppliers to be distributed in specific projects. This solution improves the workflow in the site and consequently reduces time and material stocks.

To increase the reliability of the SCM, Fischer and Kunz[8] have used information technology (IT) applications through Virtual Design and Construction (VDC) simulation of Product, Process and Organization (POP). This solution allows simulating how the actual project will be happening, improving visibility management during SCM. This method faces challenges in real-time management of POPs.

To ensure a complete solution to the constraints of communication, reliability and visibility in the construction of SCM, S. Cho (2010)[9] introduced an integrated system combining the methods of LPS, VDC and real time. The aim of his research was to increase upstream and downstream SCM relationships between demand and supply with a focus on the reduction of waiting times between different parts of the chain (customers and suppliers). The concept of this methodology is based on the digitization of each step. First, goods information is read through RFID tags and bar codes. Then, coloring takes place depending on the condition of the goods and quantification (method VDC) with registration in the database of the ERP. The different steps of reception, storage and exploitation are escorted by a real time data broadcasting. The reporting is assured also in real time through web sites (LPS) where the 4D plans $(3 \mathrm{D}+$ time) and reports are updated and accessible to all the SCM members.

\subsection{Real Time Process Management}

The 21 st century digital economy fueled by globalization and information communication technology (ICT) is characterized by processing, instantly access and dissemination of information around the world. The ability for organizations to be agile and responsive became increasingly complex, rapidly changing and interconnected in the global environment. All this requires real-time management and coordination of the value chain of the company. Management in real time requires the ability to perform transactions through computer networks, the ability to react to situations with expertise and knowledge, and the ability to leverage business intelligence[3].

The notion of real-time processing activity[4] refers to the ability of a system to respond immediately on time when a transaction occurs. It is often used in the management of advanced processes.

Joseph O. Chan (2007)[3] introduced the context of "digital economy" a real-time management of the value chain (Real Time Value Chain Management RTVM). It presents the real-time features for each activity of the value chain. His proposed model for real-time management is enabled by technologies in e-business (E-Business Value Chain Management EBVCM), Knowledge Management (Knowledge Value Chain Management KVCM) and Business Intelligence (Business Intelligence Value Chain Management BIVCM). The model of Joseph is an improvement of the one proposed by Chan, J.O.[6]. It resulted from an intersection between the three dimensions covering all logistics activities of a company by the management and real-time control of operations (production activities, storage), supplies and services (CRM customer relationship).

\subsection{Technology and the Industries Challenges}

The evolution of technologies in industries has gone through the steps overlap discrete transaction automation, improved functional activities, cross -activity integration, the integration of value chains and the optimization of the various activities in the value chain in real time[5].

Wireless Manufacturing (WM) presents the latest industry technology (Advanced Manufacturing Technology AMT). In fact, George Q. Huang, YF Zhang and PY Jiang[12] propose a solution to face the bottleneck of capturing and collecting real time field information.. This structure allows the involvement of the supply chain members, wireless management and real-time activity. The proposed architecture has three layers: Enterprise Data Repository, Enterprise Application System EAS and Application Explorer. The communication is insured by Wireless Networks.

\subsection{Wireless Communication and Process Automation}

Often, in real-time SCM, RFID and WSN technologies are introduced for the management of transportation logistics, product and raw materials inventory. Indeed, many researches in this field aim to improve the functioning of the supply chain. The objective is to manage 
in time the flow of information and material through nodes and wireless communication systems.

In this context, Sensor Scheme project[10], introduces wireless technologies (WSN and RFID) in the automation of SCM. The "cold chain system" solution is based on the monitoring and control of products conditions in real time during transport and storage (temperature, humidity, expiration, etc.). To ensure the real-time management, the authors used the Zigbee protocol for communication between WSN. As far as, L. Gomez, M. Laurent, Ethmane El Moustaine[11, 1] proposed an integration solution between RFID and WSN to provide a complete solution for the identification, control, and monitoring of the entire chain of transport and storage of materials. These solutions are based on heterogeneous network architecture with constraints and challenges in energy, maintenance and flow with limitations in activities of transport and storage.

\subsection{Information System}

Throughout the supply chain, the interaction between the members and the sharing of data is done by the ERP system. When using wireless technology for real-time monitoring of physical products and information flows the workflow in the ERP ${ }^{1}$ becomes limited. Indeed, only officials are involved in a collaborative way in this chain. In addition, several constraints are reported for these solutions[13]:

Lack of flexibility that frequently requires adapting to all or part of the solution process, and not the reverse.

Complex Settings requiring the use of specific external expertise and extended deployment delays.

Additional application development in order to further integrate these solutions with third-party applications.

The required presence of a functional administrator responsible for the proper functioning of the solution.

In addition, internal collaboration is limited, which leads to a partial automation (Workflow) of documents and information. Improvements and automation of processes are major concerns of leaders, policy makers and managers in business management. These are indeed key levers, not only to gain business agility and efficiency in decisionmaking, but also to better control or reduce costs (processing a folder, a request, customer care, complex events, complaints...). Similarly, the integration of more advanced activities within the business ecosystem of partners, customers or suppliers, creates interdependencies that require a review of approaches[14]. Indeed, ICT tools are the core of optimization and improvement of the internal and external performance of companies[15].

The modeling of SCM is based on the existing activities and processes. Thus, a common database and a shared management integrated business solution remains frozen with limited flexibility. In addition, the total management of SCM is not assured because of the reduced involvement of the different actors. The different SCM solutions are almost

\footnotetext{
${ }^{1}$ ERP: Enterprise Resources Planning
}

sharing the global structure[9, 12] which is shown in figure1.

Limitation in storage and tracking transport.

Dependency of activities (delay, performance).

Information System (ERP) limited to the standards modules and lack of flexibility.

SC Modules focus only on the transports, storage and delivery which present a reduced functionality in internal process management.



Figure 1. Classic SCM Modeling

$\tau_{\mathrm{i}}$ : delay of activity processing

$\alpha_{\mathrm{i}}$ : internal coefficient of performance

$\square_{\mathrm{i}}$ : Process

6: Performance

For $\mathrm{n}$ activities in total SCM, the global performance 6 depends on $\tau, \alpha$, and the number of processes $\square$.

$$
\text { ? }=\sum \alpha \mathrm{l} . \tau \mathrm{l} \text {. }
$$

For an enormous number of processes, the total activity performance of SC decreases.

The dynamic modeling of logistics activities and the interaction of SCM and Internet is the purpose of many researches. However, W.M.P. van der Aalst[21] introduced a framework based on a hierarchical high-level Petri net model called ExSpect. This method is based on a complete taxonomy of the goods and information flows inside a logistic system. Based on this taxonomy, a toolkit of standard logistical components was developed that can be combined graphically, thus yielding an ExSpect specification which can be used to analyze the logistic system under consideration.

\section{State of the Art}

In the context of the digital economy and business intelligence, a solution leading to a better internal processes and external collaboration (customers and suppliers) to the SCM of a company remains a big challenge. However, researchers have used ICT to monitor and control real-time data and physical flows. The scope is limited to the management of storage and transport which leads to a reduced integration in the company's management system. In addition, working on the real-time control of processes is based on ERP for which access is limited to selected actors in the supply chain. This leads to a reduced collaboration and a remarkable assignment of real-time total management process. In fact, the inflexibility of theses ERP systems is 
often pointed as being a limiting factor to their use. Organizations that adopt these types of systems end up having the processes designed in a standard form, just because the implemented system requires so[19].

The effectiveness of the ERP has become progressive squalor that doesn't meet the expectations of industrial realtime management of all business processes. However, the integration of RFID and WSN technologies in ERP is limited and the data flow is not adequately insured.

\section{Modeling of Solution}

To circumvent this problem and manage all processes and improve internal and external collaboration in the supply chain, we propose a system based on Ad hoc mobile technology to collect and disseminate real-time information. Thanks to a dynamic architecture management solution that fits any business need to manage the supply chain exact time. Communication, monitoring and control as well as involvement in the workflow are provided for all members of the chain. The objective is to minimize human errors and improve the automation of all industries processes.

With the significant changes and the increasing penetration of tablets and smartphones in the industry[16], it is essential to use these platforms to develop the ERP distributed with a decentralized architecture. This is to replace the existing conventional solutions and improve more realization attempts of mobile business applications.

\subsection{Co-Modeling of Real Time SCM (RTSCM)}

To obtain a dynamic solution, the proposed solution is based on flexible SCM and the defragmentation of all internal and external activities of the business processes and sub-processes.

Then, we propose a dynamic model of management involving different parts each module covers an activity. The interaction is ensured through a real-time communication at different managerial levels. The involvement of stakeholders is ensured through the IS in mobile and dynamic leveraging ad hoc technology and wireless networks.

To ensure this, we will adopt a new approach in comodeling of SCM and IS based on:

Designing a structure that meets all the constraints for a solution of co-modeling information system and management system.

Developing an application based on a flexible architecture to meet the dynamic constraints and collaborative logistics internally and externally.

\subsubsection{Supply Chain Management Modeling}

The total collaborative SCM shown in figure 2 is based on:

Involving the external parties (suppliers, customers, etc.).

Any modification (adding, changing or removing processes) can happen without affecting the system

Each process model contains a flexible configuration
Each process operates independently and changes data with other processes.

A SC manager coordinates between all processes and exchanges data in real time with all other actors.

The non-functioning of one process does not affect the functioning of the system.

All actors can detect problems of each process in the system and can improve the time of resolving defects.

This approach must be followed by a distributed IS.

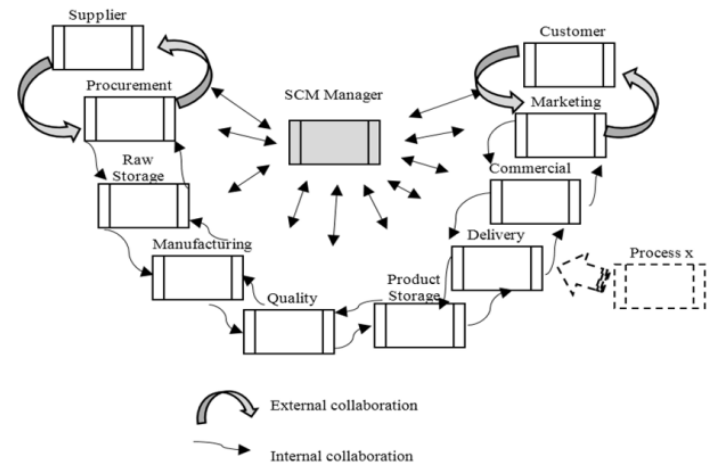

Figure 2. Proposed SCM Modeling

\subsubsection{Information System Modeling}

The IS that responds to the proposed approach is a fully integrated solution. It incorporates the internal and the external actors in a could computing system. The RFID and the WSN are deployed to collect and transmit data around the specific system configuration of the distributed architecture.

The storage of all data can be in local and/or in a could data base.

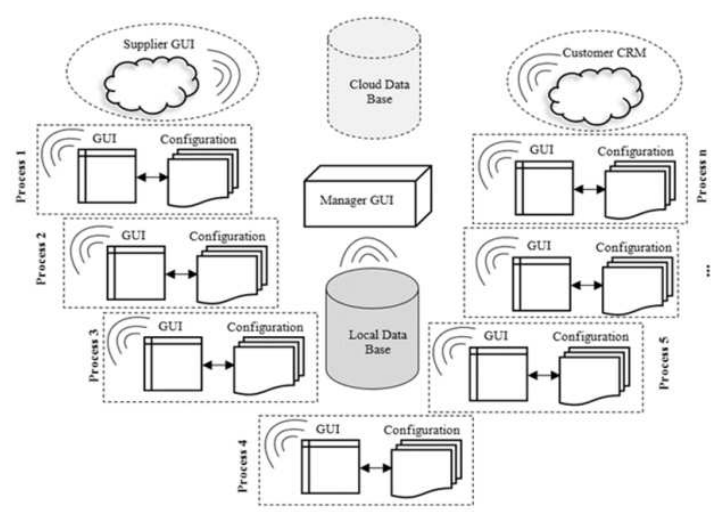

Figure 3. Proposed IS architectureModeling

\subsection{Data Foundation of Co-Modeled RTSCM.}

The proposed model of total real time supply chain management TRTSCM, figure 4 , is built by the comodeling of IS and flexible SCM. Each process (SC) is modeled as a module and (IS) is divided to sub-processes (components). All the actors and members of global SCM (internal and external) are implicated. The real time communication is insured by:

WSN/RFID with Zigbee protocol for the local 
communication.

Distributed smart phones/tablets within internet/intranet communication.

The workflow is automated between all actors and any modification that occurs must be shared at the time to the target member.

All internal processes of companies (manufacturing, logistics, inventory, quality ...) can be modeled and integrated.

The system provides a full management of all levels and involves real time decision making.

To avoid the complexity of this approach, we optimize each module and its components to obtain efficiency and reliability. Each conceptual process must cover an exact activity maintaining the inter-communication within the system activities.

The communication architecture takes into account an adaptive mechanism for wide area and large scale between the sensors to the gateway. This presents an axis for the future work.

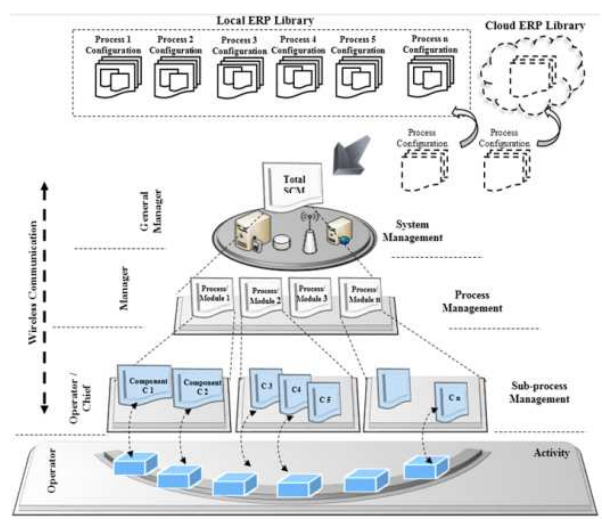

Figure 4. Structure of total real time SCM

The information and data flow are assured across all levels which enhance the collaborative management and improve the competitiveness and the total quality of the management system.

\section{Conclusion and Future Work}

The automation and the integration of all processes in the same management system that replaces the classic ERP is the core of our contribution. Our approach is based on the co-modeling of information system and the total supply chain management. The purpose is to improve the competitiveness and the quality of service by real time management. Thus, we focused on the implication of all stakeholders by using the proposed solution.Our approach is characterized by a flexible and dynamic configuration.

This work should be followed by a review and analysis of a set of solutions for the implementation and designing the proposed models. In addition, we will achieve this work by a validation of all the conceptual approach.

\section{Acknowledgments}

The authors would like to acknowledge the assistance of all who have contributed to improve the quality of this work.

\section{References}

[1] L. Zhang, Z. Wang (2006), « Integration of RFID into Wireless Sensor Networks: Architectures, Opportunities and Challenging Problems». Proceedings of the Fifth International Conference on Grid and Cooperative Computing Workshops (GCCW'06) -2006, p 1.

[2] Pascal ROOS. (2003), «The information systems, performance levers logistics company » «Les systèmes d'informations, leviers de la performance logistique de l'entreprise ». Creg.ac-versailles.fr 2003.

[3] Joseph O. Chan (2007)," Real-Time Value Chain Management". Communications of the IIMA. Volume 7 Issue 32007 p-79-88.

[4] Ralston, A. \& Reilly, E.D. (1983). "Encyclopedia of Computer Science and Engineering", 2nd Edition. New York, NY: Van Nostrand Reinhold Company Inc.

[5] Porter, M.E. (2001). "Strategy and the Internet". Harvard Business Review, 79(3), 62-78.

[6] Chan, J.O. (2006). "The Anatomy of Real-Time CRM". Communications of International Information Management Association. 6(1), 115-124.

[7] Arbulu, Roberto. and Ballard, Glenn. (2004). "Lean Supply Systems in Construction." Proceedings of the 12th Annual Conference for International Group for Lean Construction, Carlos Formoso and Marton Marosszeky. 2004 p9.

[8] Martin Fischer and John Kunz (2004), “The Scope and Role of Informationtechnology in Construction". CIFE Technical Report \#156 february 2004 Stanford University, p 3-16.

[9] Sangwoo Cho (2010), "Real-Time SCM using VDC and Lean". CIFE, Stanford University, April 2010, p12

[10] L. Evers P.J.M. Havinga J. Kuper M.E.M. Lijding N. Meratnia(2007) "SensorScheme: Supply Chain Management Automation using Wireless Sensor Networks". European 6th Framework Programme as part of the projects CoBIs (IST-2004270) and e-SENSE (IST-4-027227). 1-4244-08261/07/\$20.00 (C) 2007 IEEE $p$ 448-455.

[11] Laurent Gomez, Maryline Laurent, Ethmane El Moustaine (2011), « Integration of RFID and Wireless Sensor Networks into a Supply Chain Management System". SENSORCOMM 2011 : The Fifth International Conference on Sensor Technologies and Applications, 2011, p 393-398.

[12] George Q. Huang, YF ZhangPY Jiang (2008), "RFID-based wireless manufacturing for real-time management of job shop WIP inventories". Springer-Verlag London Limited 2008, p 753-764.

[13] MARKESS International. (2011), «Industries expectations for integrated ERP solutions face new challenges ", «Attentes des entreprises pour les solutions de gestion intégrée ERP/PGI face aux nouveaux enjeux». Paris, France, 
Septembre (2011) p 3.

[14] MARKESS International. (2011),«Improvement and automation ofbusiness processes ", "Amélioration \& automatisation des processus d'entreprise». Paris, France, Mars (2011) p 2. www.markess.fr.

[15] MARKESS International. (2010), «The ICT, levers development forlocal authorities », «Les TIC, leviers de développement pour les collectivités territoriales». Paris, France, Décembre (2010) p 3. www.markess.fr.

[16] MARKESS International. (2011), «Mobile business applications on smartphones and digital tablets », «Applications mobiles professionnelles sur Smartphones et tablettes numériques». Paris, France, (Octobre 2011) p 6. www.markess.fr.

[17] PMP Research. «ERP LEADS FIGHT AGAINST INFORMATION OVERLOAD» p1. http://www.pmp.co.uk/

[18] Sergey V. Zyko (2002).“Enterprise Resource Planning Systems: the Integrated Approach" p1. ITERA International
Group of Companies, Moscow, Russia.

[19] Paula Serdeira Azevedo, Mário Romão, Efigénio Rebelo (2012). "Advantages, Limitations and Solutions in the Use of ERP Systems (Enterprise Resource Planning) - A Case Study in the Hospitality Industry". CENTERIS 2012 Conference on ENTERprise Information Systems / HCIST 2012 - International Conference on Health and Social Care Information Systems and Technologies. Procedia Technology 5 ( 2012 )264-272 .

[20] Marc Schumacher and Dustin Gillespie (2005). "RFID: ERP as a Critical Component”. P7. MGMT 579: ERP.

[21] W.M.P. van der Aalst.(1992) "Logistics: A Systems Oriented Approach". P1 Department of Mathematics and Computing Science. Eindhoven University of Technology.The Netherlands.

[22] Cristina Giménez, Helena R. Lourenço. «E-Supply Chain Management: Review, Implications And Directions For Future Research “. 